Please do not remove this page

RMIT

UNIVERSITY

\title{
Adaptive geometric features based filtering impulse noise in colour images
}

Xu, Zhengya; Qiu, Bin; Wu, Hong Ren; Yu, Xinghuo

https://researchrepository.rmit.edu.au/esploro/outputs/9921863971801341/filesAndLinks?institution=61RMIT_INST\&index=null

Xu, Z., Qiu, B., Wu, H. R., \& Yu, X. (2008). Adaptive geometric features based filtering impulse noise in colour images. Proceedings of the 2008 IEEE 10th Workshop on Multimedia Signal Processing, 247-251. https://doi.org/10.1109/MMSP.2008.4665083

Published Version: https://doi.org/10.1109/MMSP.2008.4665083

Repository homepage: https://researchrepository.rmit.edu.au

(C) 2008 IEEE

Downloaded On 2023/04/26 23:55:37 +1000

Please do not remove this page 


\title{
Adaptive Geometric Features Based Filtering Impulse Noise in Colour Images
}

\author{
Zhengya $\mathrm{Xu}^{\# 1}$, Bin Qiu ${ }^{* 2}$, Hong Ren $\mathrm{Wu}^{\# 3}$ Xinghuo $\mathrm{Yu}^{\# 4}$

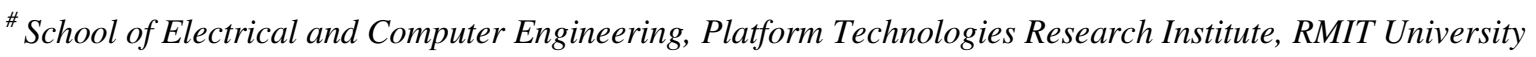 \\ VIC 3001, Australia \\ zhengya.xu@rmit.edu. au \\ ${ }^{3}$ henry.wuermit.edu. au \\ ${ }^{4}$ x.yuermit.edu.au \\ *Faculty of Information Technology, Monash University, \\ VIC 3800, Australia \\ bin.qiueinfotech.monash.edu.au
}

\begin{abstract}
An Adaptive geometric features based filtering (AGFF) technique with a low computational complexity is proposed for removal of impulse noise in corrupted color images. The effective and efficient detection is based on geometric characteristics and features of the corrupted pixel and/or the pixel region. A progressive restoration mechanism is devised using multi-pass non-linear operations. Through extensive experiments conducted using a wide range of test color images, the proposed filtering technique has demonstrated superior performance to that of well-known benchmark techniques, in terms of objective measurements, the visual image quality and the computational complexity.
\end{abstract}

\section{INTRODUCTION}

Non-linear filtering techniques have been extensively researched in the last decade due to their importance in restoration of noise corrupted color images. The median filter is usually used to remove impulse noise [1], [14]. The most well known vector filters for color image denoising include the vector median filter (VMF) [2], and the directional distance filter (DDF) [3].

Unlike the additive noise that contaminates all image pixels, the impulse noise destroys only a small portion of an image and leaves other pixels noise free. Detection based vector filtering techniques such as the adaptive vector median filter (AVMF) [6], the adaptive vector LUM smoother (AVLUM) [8], modified weighted vector median filter (MWVM) [9], and the adaptive selection center weighted vector direction filter (ACWVDF) [10], were specially designed to remove the impulse noise from color images. They utilize a series of weighted median vector filters to perform the binary noise detection and switch the output between an identity filter and a weighted median vector filter according to the detection results.

In this paper, a fast adaptive geometric features based filtering technique (AGFF) with a low computational complexity is proposed for restoration of digital color images corrupted by the impulse noise. This new technique uses a set of novel noise detection criteria for detection of the corrupted pixels, which are based on two-dimensional geometric and dimension features of the noisy pixel or the noisy region of the images. Based on the result of the estimation, an adaptive progressive filtering operation is employed in combination with optimized dimension and shape of processing windows. It is very useful for online applications for fast suppression of impulse noise in medium- and large-size color images.

The organization of this paper is as follows. Section II addresses the principles of the proposed filtering technique. Experimental results are presented in Section III. Final conclusions are drawn in Section IV.

\section{PRINCIPLE OF AGFF TECHNIQUE}

The fast adaptive geometric feature based filtering technique (AGFF) is introduced as follows, for the restoration of colour images corrupted by the impulse noise.

\section{A. Dimensional and Geometric Features of the Impulse Noise}

A major problem in restoration of color images to date is the destruction of detailed image structures due to inability of denoising filters to distinguish a cluster of corrupted pixels from a cluster of pixels presenting fine (detailed) image structures and the incorrect removal or modification of pixel segments. This section proposes a novel technique, which detects, exactly and efficiently, impulses in color images.

Careful examinations of a variety of color images corrupted impulse noise models reveal that most of uncorrupted pixels or pixel regions in a natural color image always demonstrate a certain degree of smoothness. This means that the color intensities of 8-neighbors[12] of a pixel always change gradually in all directions (e.g., in a smooth area), and at least gradually change in one (edge) direction (e.g., in a boundary area). In contrast with normal pixels of images, impulse noise corrupted pixels always show their features as an isolate spot or cluster by its very un-harmonic colors, shapes and sizes compared with those of its neighborhood. It is observed that almost all impulses only have sharp step edges and, in contrast, almost no uncorrupted object has this type of edges in its vicinity. The shapes of the noise regions may be an isolated point, a short thin line, a cross of two short thin lines or other small round-shaped blocks. In other words, the shape and the 
size of impulse noise corrupted pixels depend on the noise ratio.

According to the above observation and analysis of color, shape and sizes of impulse noise corrupted pixels/regions, and the type of edges which form the borders of the noise regions, a novel impulse noise detection method is proposed here based on two dimensional geometric features of the impulses, in stead of the one dimensional rank ordered statistical information used by other well-known filtering techniques, to determine more exactly and efficiently if each pixel in a color image is corrupted or clean. One of the geometric properties of the impulse noise is the edge feature of its boundary. An edge can be defined as a local discontinuity in illumination function and the edge orientation is defined as edges of an octagonally shaped object whose amplitude is higher or lower than its background [12]. Therefore, the proposed criteria for identifying the edge feature around the pixel are based on two types of derivatives, which are approximated by differences in digital color images. Given that $C \equiv\left\{c=\left(c_{1} c_{2}\right) \mid 1 \leq c_{1} \leq H, 1 \leq c_{2} \leq W\right\} \quad$ denote the pixel coordinates of a color image, where $H$ and $W$ are the height and width of the image, respectively. If $\mathbf{x}(c)=\left[x_{R}(c), x_{G}(c), x_{B}(c)\right]^{T}$ is the illumination function of a color image, the two special types of derivative are denoted as $\partial x(c) / \partial c^{a}$ and $\partial x(c) / \partial c^{d} . \partial x(c) / \partial c^{a}$ is defined as follows,

$$
\left\{\begin{array}{l}
\partial x(c) / \partial c_{1}^{a}=x[i, j]-x\left[i-n_{a}^{1}, j\right] \\
\partial x(c) / \partial c_{2}^{a}=x[i, j]-x\left[i, j-n_{a}^{2}\right] \\
\partial x(c) / \partial c_{3}^{a}=x[i, j]-x\left[i+n_{a}^{3}, j\right] \\
\partial x(c) / \partial c_{4}^{a}=x[i, j]-x\left[i, j+n_{a}^{4}\right]
\end{array}\right.
$$

where, $n_{a}>0$, and the default value of $n_{a}$ is 1 .

When a derivative is only considered in the diagonal direction, $\partial x / \partial c^{d}$ is defined as follows,

$$
\left\{\begin{array}{l}
\partial x(c) / \partial c_{1}^{d}=x[i, j]-x\left[i-n_{d}^{1}, j-n_{d}^{1}\right] \\
\partial x(c) / \partial c_{2}^{d}=x[i, j]-x\left[i+n_{d}^{2}, j-n_{d}^{2}\right] \\
\partial x(c) / \partial c_{3}^{d}=x[i, j]-x\left[i-n_{d}^{3}, j+n_{d}^{3}\right] \\
\partial x(c) / \partial c_{4}^{d}=x[i, j]-x\left[i+n_{d}^{4}, j+n_{d}^{4}\right]
\end{array}\right.
$$

where, $n_{d}>0$, and the default value of $n_{d}$ is 1 .

The denoted derivatives, $\partial x(c) / \partial c^{a}$ and $\partial x(c) / \partial c^{d}$ will be used to measure the edge feature (sharpness) and other geometric properties to determine whether the center pixel at $c(i, j)$ is corrupted or not in the proposed filtering technique.

In detecting and removing impulse noise, a filter can make different types of mistakes. Type I error (miss) occurs when there is a corrupted pixel, which the filter does not detect. Type II error (false alarm) happens when the filter detects an impulse noise pixel, which is actually clean. When the filter removes an impulse noise and replaces it with a value determined by a certain restoration strategy, Type III error (over- or under-correcting error) is defined as the difference between the resultant value after restoration process and the true pixel value as the noise-free pixel was.

The proposed technique, similar to other well-known benchmark techniques and the so-called "switching" filters $[5,6,11,18]$, consists of two components, i.e., impulse detection and impulse removal.

A key component of the proposed filtering technique is a novel impulse detection scheme based on the two dimensional geometric information of the corrupted pixels. The novel criteria used by the proposed filtering technique for noisy pixel detection are based on a combination of the twodimensional edge, geometric and size features of the noisy $\mathrm{pixel} /$ region in the images. They depart from traditional noise detection techniques used by other existing filters $[5,6,2,13]$, which only use some properties of the edge of a noisy pixel or one-dimensional rank ordered statistical information around the noisy pixel. First, we define the edge feature-identification threshold, $T_{e}$, which represents the value of a derivative to distinguish the sharp step edges from other types of edges. Since very short thin lines usually form the impulse noise pixels, the length of a line is also used as a feature to distinguish a short noise line from a fine line in color images. The length threshold may be defined as $T_{l}$ according to the noise ratio.

Second, in terms of the pixel coordinates of a color image, $C$, a set of corrupted pixels is defined as

$$
\begin{array}{r}
S_{l}=\left\{c \mid\left(\left(\partial x(c) / \partial c^{a}<\left(-T_{e}\right)\right) \wedge\left(\partial x(c) / \partial c^{d}<\left(\left(-T_{e}\right)\right)\right) \vee\right.\right. \\
\left(\left(\partial x(c) / \partial c^{a}>T_{e}\right) \wedge\left(\partial x(c) / \partial c^{d}>T_{e}\right)\right), N_{d}=\left\{1,2,3, \ldots T_{m}\right\},
\end{array}
$$

$\left.n_{d}^{i} \in N_{d}, i=1, \ldots, 4\right)$

where $T_{m}=\left(T_{l}+1\right) / 2$. The default value of $T_{m}$ is 2 . This corrupted pixel set includes individual impulse pixels, slant noise lines with one-pixel width and the pixels of the lines only adjacent to each other in diagonal direction within the defined length of $T_{l}$.

Third, a set of corrupted pixels, which include individual impulse pixels, straight noise lines with one-pixel width and the pixels of the lines being only 4-connected[12] to each other within the defined length of $T_{l}$, is defined as

$$
\begin{aligned}
& S_{2}=\left\{c \mid\left(\left(\partial x(c) / \partial c^{a}<\left(-T_{e}\right)\right) \wedge\left(\partial x(c) / \partial c^{d}<\left(-T_{e}\right)\right)\right) \vee\right. \\
& \left(\left(\partial x(c) / \partial c^{a}>T_{e}\right) \wedge\left(\partial x(c) / \partial c^{d}>T_{e}\right)\right), N_{a}=\{1,2,
\end{aligned}
$$

$\left.\left.3, \ldots T_{m}\right\}, n_{a}^{i} \in N_{a}, i=1, \ldots, 4\right\}$

where $T_{m}=\left(T_{l}+1\right) / 2$. The default value of $T_{m}$ is 2 .

Finally, a set of corrupted pixels, which include noisy pixels/regions within 3-pixel width in any direction except the noisy pixels c $\in S_{1} \cup S_{2}$, is defined as $S_{3}$.

If $S=\left\{c \mid\left(\left(\partial x(c) / \partial c^{a}<\left(-T_{e}\right)\right) \wedge\left(\partial x(c) / \partial c^{d}<\left(-T_{e}\right)\right)\right) \vee\right.$ $\left(\left(\partial x(c) / \partial c^{a}>T_{e}\right) \wedge\left(\partial x(c) / \partial c^{d}>T_{e}\right)\right), n_{d}=n_{a}=2$ or 3$\}$

where the default value for $n_{d}$ and $n_{a}$ is 2 in (5). Thus, $S_{3}$ can be represented as

$$
S_{3}=S-\left(S_{1} \cup S_{2}\right)
$$


where $T_{m}=2$ for $S_{1}$ and $S_{2}$ in (8). Since an impulse noise ratio $p_{I}<1, \bigcup_{i=1}^{n} S_{i} \subset \mathrm{C}$, where $n=3$, in the current case. $T_{e}$ in (3), (4) and (5) could be set in different values.

Since the shapes and the sizes of corrupted pixels depend on the noise ratio, and the restoration of the corrupted pixels requires the statistical information about the noise density, the estimation of noise ratio and noise type is important in order to minimize both Type I and Type II errors. The criteria for classifying the degree of impulse noise in the proposed filtering technique are the ratio and the size of the largest noise corrupted region, and the vales of corrupted pixels.

The strategy of the progressive restoration for the proposed filtering technique is first to restore corrupted individual pixels or noise regions of small size. If it made either Type II or Type III error, it should not introduce any new impulse noise region of bigger size than the existing ones. Then, further operations are carried out around big noise corrupted regions, to restore areas of the images associated with big noise regions more reliably.

In order to make use of merits of the median filter and to avoid its drawbacks (causing a number of artifacts for uncorrupted pixels) [14], a detection scheme described in this section is employed before the median filtering for restoration. As a result, the proposed restoration method based on the restricted median can keep the image unchanged when the filter processing window moves across the uncorrupted image details.

A novel progressive multi-pass filtering algorithm/process with low computational complexity is proposed, in order to implement the principles of proposed filtering technique.

The restoration technique used in this work is based on the modified median where the destruction of uncorrupted images (i.e., Type II error) increases with the increase of the processing window size, while decreases with the increase of the edge feature threshold $T_{e}$. In order to achieve the best performance of the proposed filter, in terms of both visual quality and objective measurements, the design of the processing windows has to depend on the shapes and sizes of corrupted pixels/pixel regions.

A principle for designing the following operations is to use as small a size of the windows and as less a number of the passes as possible, as long as the impulse noise can be removed (to ensure perceptual image quality). The number of passes was determined to remove noise region based on the worst case scenario within the estimated maximal size of the noise region. Actually, the adaptive filter is very robust and tolerant to the estimation deviations for impulse noise ratio of corrupted images.

For the proposed filter (AGFF), in contrast with other filters, only comparison and addition/subtraction operations are involved, and the computational complexity of the AGFF is mainly dependent on the restoration operations. Although the proposed filter uses multi-pass operation and the time consumed by the filter is dependent on the noise ratio, the computational complexity of its algorithm is actually very low.

\section{EXPERIMENTS}

Without losing generality, the recommended the definition of the corrupted pixel sets in color images were tested by experiments using typical test images, which included different types of real-life images [17]. Table I presents the experimental results for detection of random impulse noise using $S_{1}, S_{2}$ and $S_{3}$ on a variety of original clean (without corruption) real life images, which include well-known standard test images such as, Airplane, Boats, Flower, Girl, Goldhill, Moon, Pen, Soccer, Zelda, and Yacht with an image resolution of $500 \times 362$ or $512 \times 512$ or $787 \times 576$ or $720 \times 576$ or $768 \times 512$ or $1986 \times 1986$ pixels. In Table 1, FA (false alarm) stands for Type II error, and $\operatorname{FA}\left(S_{1}\right), \operatorname{FA}\left(S_{2}\right)$ and $\operatorname{FA}\left(S_{3}\right)$ denote false alarm ratios (the number of false alarm to the number of pixel for the tested color image) by $S_{1}, S_{2}$ and $S_{3}$, respectively. The false alarming ratios are very low for the test images, especially for the high resolution color images.

The proposed filtering technique has been evaluated by an extensive range of tests and its performance is compared with a number of prior-art filtering techniques in the area of removing impulse noise from color images. Several objective criteria are used in the tests to measure the distortion in restored images. The objective criteria include the Mean Square Error (MSE) and the Mean Absolute Error (MAE) defined in the RGB color space $[4,10]$, and the Normalized Color Difference (NCD) [4,10] which measures the color distortion in perceptual uniform CIELUV color space.

All impulse corruptions were generated according to the noise model in [11], using the random impulse noise or the salt-and-pepper noise, and a noise ratio $p_{I}[11]$ varied from $0 \%$ to $20 \%$. The evaluation of impulse suppression was conducted using three 24-bit RGB images [17], "Lena", "Parrots" and "Peppers", with image resolutions of 256x256, $512 \times 512$, and 1536x1024 pixels, respectively, which have been widely used by prior-art impulse filtering techniques due to their representative color characteristics and image structure.

The filters are used in the impulse suppression tests includes the state-of-the-art techniques recently developed for the impulse and the mixed noise suppression, including AVMF, MWC[16], SAA[19], AVLUM, ACWVDF, SCWVDF[10], ACWMF[15], SWVDF[20], HBTM[7], SAHVF[5] and PBTVM[11].

Besides the excellent objective performance measurements, the proposed filter also achieved a consistently better performance in perceptual image quality than other impulse filtering techniques. Figures 1 have demonstrated the performance of the proposed AGFF compared with other typical and state-of-the-art techniques. A test image "Lena" with $20 \%$ random impulse corruption generated by noise model in [11] was selected to reveal the detail preservation capability of the proposed filter (see Fig.1) 
TABLE I

THE PERFORMANCE OF THE PROPOSED THE DEFINITION OF THE CORRUPTED PIXEL SETS FOR DETECTING RANDOM IMPULSE NOISE IN A VARIETY OF ORIGINAL COLOR IMAGES

\begin{tabular}{l|lllllllllll}
\hline Images & Airplane & Boats & Flower & Girl & Goldhill & Parrots & Moon & Pen & Soccer & Yacht & Zelda \\
\hline \hline $\operatorname{FA}\left(S_{1}\right) 10^{-3}$ & 0.009 & 0.003 & 0.001 & 0.001 & 0.034 & 0.029 & 0.062 & 0.000 & 0.003 & 0.007 & 0.003 \\
\hline $\operatorname{FA}\left(S_{2}\right) 10^{-3}$ & 0.020 & 0.051 & 0.019 & 0.018 & 0.119 & 0.076 & 0.122 & 0.025 & 0.094 & 0.104 & 0.012 \\
\hline $\operatorname{FA}\left(S_{3}\right) 10^{-3}$ & 0.056 & 0.007 & 0.008 & 0.002 & 0.047 & 0.026 & 0.081 & 0.056 & 0.065 & 0.064 & 0.022 \\
\hline
\end{tabular}

TABLE II

The impulse suppression performance of the proposed AGFF filter compared with other techniques.

(a) Color image Peppers corrupted by different levels of random impulse[11].

\begin{tabular}{l|lll|lll|lll}
\hline \multirow{2}{*}{ Filters } & \multicolumn{3}{|c|}{$5 \%$} & & \multicolumn{3}{c|}{$10 \%$} & \multicolumn{3}{c}{$20 \%$} \\
\cline { 2 - 9 } & MSE & MAE & NCD & MSE & MAE & NCD & MSE & MAE & NCD \\
\hline \hline SAA & 17.5 & 0.511 & 0.0045 & 33.5 & 0.829 & 0.0086 & 73.0 & 1.782 & 0.0185 \\
AVLUM & 22.1 & 0.465 & 0.0042 & 44.6 & 0.926 & 0.0083 & 93.2 & 1.875 & 0.0178 \\
HBTM & 19.3 & 0.466 & 0.0039 & 36.4 & 0.880 & 0.0078 & 73.4 & 1.702 & 0.0172 \\
AVMF & 25.6 & 0.556 & 0.0045 & 38.8 & 0.999 & 0.0089 & 89.2 & 1.982 & 0.0189 \\
ACWVDF & 21.1 & 0.693 & 0.0072 & 59.7 & 1.212 & 0.0127 & 180.2 & 2.774 & 0.0296 \\
SCWVDF & 20.5 & 0.666 & 0.0070 & 52.8 & 1.110 & 0.0120 & 145.5 & 2.361 & 0.0254 \\
SWVDF & 18.1 & 0.962 & 0.0142 & 43.1 & 1.381 & 0.0196 & 132.5 & 2.615 & 0.0314 \\
PBTVM & 16.8 & 0.476 & 0.0044 & 32.5 & 0.869 & 0.0082 & 65.4 & 1.652 & 0.0167 \\
SAHVF & 29.8 & 0.946 & 0.0074 & 48.8 & 1.240 & 0.0104 & 74.9 & 2.191 & 0.0171 \\
\hline AGFF & 18.0 & 0.451 & 0.0041 & 30.2 & 0.804 & 0.0054 & 45.9 & 1.320 & 0.0088 \\
\hline
\end{tabular}

(B) COLOR IMAGE PARROTS CORRUPTED BY DIFFERENT LEVELS OF SP(SALT-AND-PEPPER) IMPULSE.

\begin{tabular}{l|lll|lll|lll}
\hline \multirow{2}{*}{ Filters } & \multicolumn{3}{|c|}{$5 \%$} & \multicolumn{3}{c|}{$10 \%$} & \multicolumn{2}{c}{$20 \%$} \\
\cline { 2 - 10 } & MSE & MAE & NCD & MSE & MAE & NCD & MSE & MAE & NCD \\
\hline \hline AVMF & 2.659 & 0.540 & 0.0004 & 3.234 & 0.614 & 0.0005 & 5.16 & 0.769 & 0.0008 \\
DDF & 3.925 & 0.609 & 0.0004 & 4.496 & 0.668 & 0.0005 & 7.265 & 0.812 & 0.0008 \\
HBTM & 0.586 & 0.061 & 0.0001 & 1.026 & 0.121 & 0.0002 & 2.434 & 0.255 & 0.0004 \\
MWC & 2.731 & 0.612 & 0.0004 & 2.522 & 0.393 & 0.0007 & 4.292 & 0.517 & 0.0011 \\
\hline FAGFF & 0.346 & 0.040 & 0.0001 & 0.623 & 0.081 & 0.0002 & 1.368 & 0.169 & 0.0004 \\
\hline
\end{tabular}

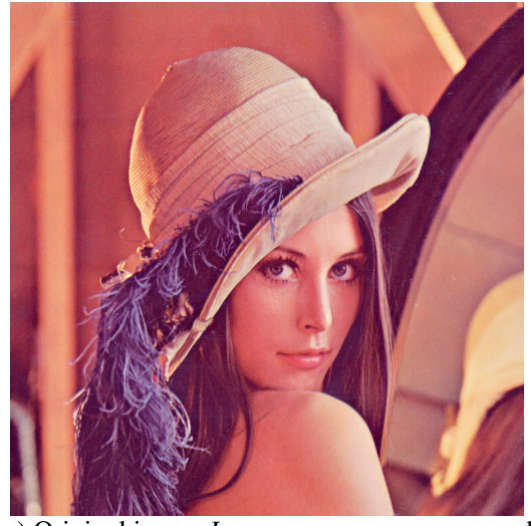

a) Original image Lena

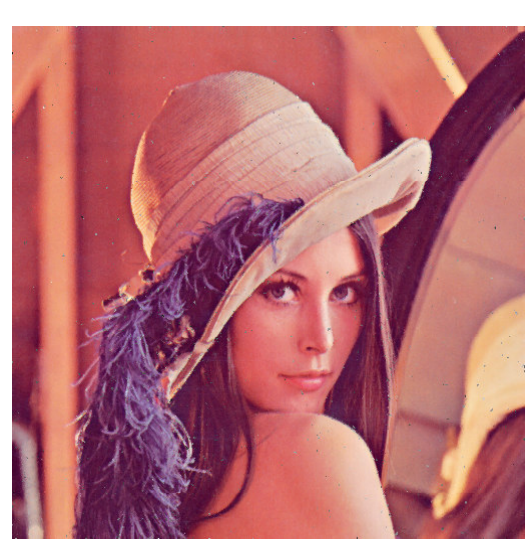

d) HBTM output

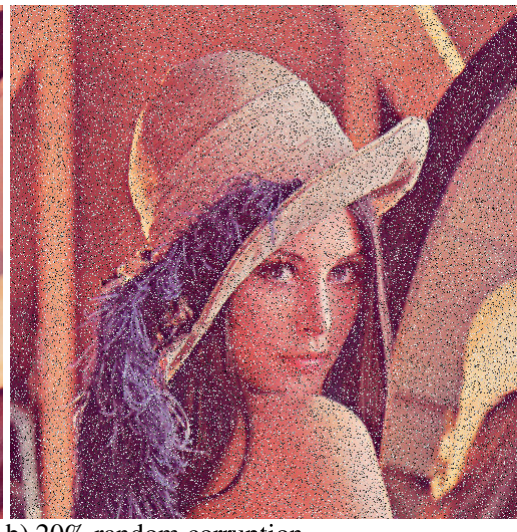

b) $20 \%$ random corruption

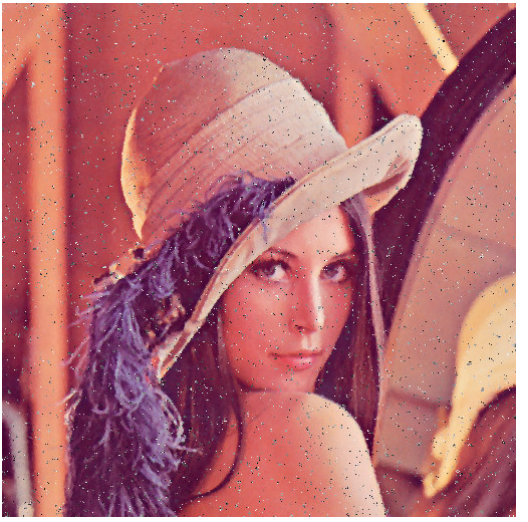

c) DDF output

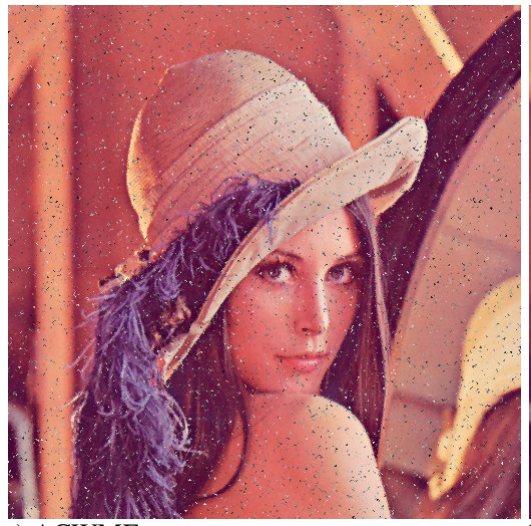

e) ACWMF output

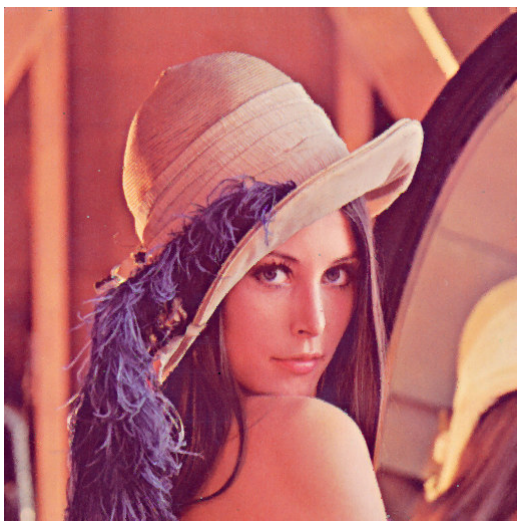

f) AGFF output

Fig. 1. The reconstruction of proposed filter compared with other techniques, where the test image "Lena" is corrupted by random impulse with $p_{I}=20 \%$ [11]. 


\section{CONCLUSION}

A geometric features based filtering technique with very low computational complexity is proposed for removing impulse noise in corrupted digital color images. The special contribution of the proposed filtering technique is its novel impulse detection, which uses two dimensional geometric features (shape and edge type) and size of the impulse pixel/pixel region, in stead of one dimensional statistical information, to identify the impulse in a more exact and efficient manner. The other novelty of the proposed filtering technique is its progressive adaptive restoration mechanism to recover the corrupted pixels step by step in a reliable way through a multi-pass process with low computational complexity. Through extensive experiments conducted using a wide range of natural color images, the proposed filtering technique has demonstrated superior performance to that of well-known benchmark techniques, in terms of standard objective measurements, visual image quality and the computational complexity, in removing different types of impulse noise commonly considered in color image restoration. The types of impulse noise include the salt-andpepper and the random impulse noise. It is very useful for online applications to suppress impulse noise especially for medium and large sized color images.

\section{REFERENCES}

[1] I. Pitas and A. N. Venetsanopoulos, Nonlinear Digital Filter Principles and Applications. Norwell, MA: Kluwer, 1990.

[2] J. Astola, P. Haavisto, and Y. Neuvo, "Vector median filter" Proc. IEEE, vol. 78, no. 4, pp. 678-689, Apr. 1990.

[3] D. G. Karakos and P. E. Trahanias, "Combining vector median and vector direction filters: the directional-distance filter" in Proc. IEEE int. Conf. Image Processing (ICIP'95), vol. 1, Washington DC. Oct. 1995, pp. 171-174.

[4] R. Lukac and K.N. Plataniotis, "A taxonomy of color image filtering and enhancement solutions," In 'Advances in Imaging and Electron Physics', (ed.) P.W. Hawkes, Elsevier, vol. 140, pp.187-264, 2006.

[5] Z. Ma, H. R. Wu, and B. Qiu, "An structure adaptive hybrid vector filter for the restoration of digital color images," IEEE Trans. Image Processing, vol. 14, no. 12, pp 1990-2001, Dec. 2005.
[6] R. Lukac, "Adaptive vector median filtering," Patt. Recogn. Lett., vol. 24, no. 12, pp. 1889-1899, Aug. 2003

[7] Z. Ma and H. R. Wu, "A histogram based adaptive vector filter for color image restoration," in Proc. IEEE ICICS-PCM'03 Singapore, Dec. 15-18, 2003, pp. 1A3.4.1-5.

[8] R. Lukac and S. Marchevsky, "Adaptive vector LUM smoother," in Proc. IEEE int. Conf. Image Processing, vol. 1, Oct. 2001, pp. 878-881.

[9] B. Smolka, M. Szczepanski, K. N. Plataniotis and A. N. Venetsanopoulos, "On the modified weighted vector median filter," in Proc. IEEE int. Conf. Digital Signal Process. Vol. 1, Santorini, Greece, July 2002, pp. 939-942.

[10] R. Lukac, "Adaptive colour image filtering based on center weighted vector direction filters," Multidimentional Syst. Signal Process, vol.15, no. 2, pp. 169-196. Apr. 2004.

[11] Z. Ma, H. R. Wu, D. Feng, "Partition based vector filtering technique for suppression of noise in digital colour images," IEEE Trans. on Image Processing, vol. 15, no. 8, pp 2324-2342, Aug. 2006.

[12] Milan Sonka, Vaclav Hlavac and Roger Boyle, "Image processing, Analysis, and Machine Vision," Brooks/Cole, 2001.

[13] S. Schute, V. D. Witte, M. Nachtegeal, D.V. Weken and E.E. Kerre, "Fuzzy two-step filter for impulse noise reduction from colour images" IEEE Trans. on Image Processing, vol. 15, no. 11, pp 3568-3579, Nov. 2006.

[14] Raymond H. Chan, Chung-Wa Ho and Mila Nikolova, "Salt-andpepper noise removal by median-type noise detectors and detailpreserving regularization" IEEE Trans. on Image Processing, vol 14 no. 10, pp 1479-1485, Oct. 2005.

[15] T. Chen and H. Wu, " Adaptive impulse detection using centerweighted median filter," Signal Processing Lett., Vol.8 no. 1pp. 1-3, Jan. 2001.

[16] E. S. Hore, B. Qiu, and H. R. Wu, "Prediction based image restoration using a multiple window configuration," Opt. Eng., vol.41, no. 8 pp. 1855-1865, Aug. 2002.

[17] http://www.hlevkin.com.

[18] P. Ng and K.K. Ma, "A Switching Median Filter with Boundary Discriminative Noise Detection for Extremely Corrupted Images", IEEE Transactions on Image Processing, vol. 15, no. 6, pp. 1506-1516, June 2006.

[19] B. Smolka, A. Chydzinski, K. Wojciechowski, K. N. Plataniotis, and A. N. Venetsanopoulos, "Self-adaptive algorithm for impulsive noise reduction in color images," Patt. Recogn., vol. 35, no 8, pp. 1771-1784, Aug 2002.

[20] R. Lukac, B. Smolka, K. N. Plataniotis, and A. N. Venetsanopoulos, "Selection weighted vector directional filter," Comput. Vis. Image Underst., vol. 94, no. 1-3, pp. 140-167, Apr. 2004. 